\title{
Evaluación larvicida de suspensiones acuosas de Annona muricata Linnaeus «guanábana» sobre Aedes aegypti Linnaeus (Diptera, Culicidae)
}

\section{Larvicidal evaluation of aqueous suspensions of Annona muricata Linnaeus «custard apple» against Aedes aegypti Linnaeus (Diptera, Culicidae)}

\author{
Miguel Bobadilla', Fátima Zavala², Manuel Sisniegas³, Gina Zavaleta ${ }^{4}$, José \\ Mostacero $^{2}$ y Luis Taramona ${ }^{1}$
}

Presentado: $02 / 03 / 2005$

Aceptado: $\quad 12 / 06 / 2005$

\section{Resumen}

EL presente trabajo evaluó la toxicidad larvicida de suspensiones acuosas provenientes de extractos etanólicos de las semillas, flores, hojas, corteza de ramas y corteza de raíces de Annona muricata L. "guanábana» sobre larvas del IV estadio de Aedes aegypti para determinar de esta manera los niveles de susceptibilidad. El mayor efecto tóxico correspondió a la suspensión de las semillas con un $100 \%$ de mortalidad a las 24 horas a $0,5 \mathrm{mg} / \mathrm{mL}$, seguidos por las flores a las 48 horas a $10 \mathrm{mg} / \mathrm{mL}$ y hojas a las 36 horas a $100 \mathrm{mg} / \mathrm{mL}$. En semillas, las concentraciones letales al $50 \%\left(\mathrm{CL}_{50}\right)$ y $90 \%\left(\mathrm{CL}_{90}\right)$ a las 48 horas de exposición fueron $0,02 \mathrm{mg} / \mathrm{mL}$ y $0,11 \mathrm{mg} / \mathrm{mL}$, en flores 3,33 y $12,16 \mathrm{mg} / \mathrm{mL}$, en hojas 8,25 y $26,87 \mathrm{mg} / \mathrm{mL}$ y en corteza de ramas 19,21 y $97,23 \mathrm{mg} / \mathrm{mL}$, respectivamente. Los resultados de las rectas probit-logarítmicas indicaron susceptibilidad de los individuos a cada suspensión, gracias a la acción de diversos principios activos distribuidos en todo el árbol.

Palabras clave: Annona muricata, suspensión acuosa larvicida, susceptibilidad, insecticida botánico.

\section{Abstract}

The aim of the present study was to evaluate the larvicidal toxicity of aqueous suspensions from ethanol extracts of Annona muricata L. "custard apple» seeds, flowers, leaves, stem bark and root bark on fourth instar larvae of Aedes aegypti L. (Diptera: Culicidae) as well as to determinate susceptibility level . The highest larvicidal toxic effect was to the seeds suspension with $100 \%$ mortality at $0,5 \mathrm{mg} / \mathrm{mL}$ from 24 hours of exposure, and flowers suspension at $10 \mathrm{mg} / \mathrm{mL}$ from 48 hours of exposure and leaves suspension at $100 \mathrm{mg} / \mathrm{mL}$ from 36 hours of exposure. In seed suspension, lethal concentrations $50 \%\left(\mathrm{LC}_{50}\right)$ and $90 \%\left(\mathrm{LC}_{90}\right)$ values were $0,02 \mathrm{mg} / \mathrm{mL}$ and $0,11 \mathrm{mg} / \mathrm{mL}$, in flowers suspensions were $3,33 \mathrm{mg} / \mathrm{mL}$ and $12,16 \mathrm{mg} / \mathrm{mL}$, in leaf suspensions were 8,25 y $26,87 \mathrm{mg} / \mathrm{mL}$ and stem bark suspensions were $97,23 \mathrm{mg} / \mathrm{mL}$, respectively. Log-dosage/probit lines indicated homogeneous responses towards the five aqueous suspensions, because of phytochemicals are distributed in each part of tree.

Keywords: Annona muricata, larvicidal aqueous suspension, susceptibility, botanical insecticide.

\section{Introducción}

Los mosquitos Aedes aegypti son vectores del dengue, fiebre hemorrágica del dengue y de la fiebre amarilla. A la fecha no se conoce una vacuna disponible para evitar dichas enfermedades y la única forma de controlarlas es mediante la erradicación o reducción a ni- veles extremadamente bajos del vector, utilizando insecticidas químicos (Rose, 2001). Por esta razón, la OPS (2001), propuso el llamado control selectivo de vectores, que involucra el uso de recursos disponibles en un determinado territorio y al alcance de la comunidad, con el propósito de adaptarlos y adecuarlos como

\footnotetext{
(1) Facultad de Ciencias. Universidad Nacional de Ancash «Santiago Antúnez de Mayolo». Perú

(2) Facultad de Ciencias Biológicas. Universidad Nacional de Trujillo. Perú

(3) Facultad de Ciencias Físicas y Matemáticas. Universidad Nacional de Trujillo. Perú

(4) Dirección Ejecutiva de Salud Ambiental. Moyabamba. Perú.

E-mail: Miguel Bobadilla: mcba_1122@yahoo.es
} 
medidas para el control de vectores. Dicha situación ha conllevado al surgimiento de recursos alternativos como los extractos naturales provenientes de plantas (Weinzierl y Henn, 1992), de potencial ayuda en el control de Enfermedades Transmitidas por Vectores (ETV) (Zimmerman, 2001; Mathur, 2003).

Muchas especies vegetales, especialmente de las Annonaceaes, han mostrado tener bioactividades, en especial la insecticida (Alali et al., 1999). Sin embargo, poco se ha investigado dentro de los programas de salud pública local y nacional, la manera de controlar los altos niveles de resistencia fisiológica alcanzados a causa de los insecticidas órganofosforados actualmente en uso (OMS, 1992). Por este motivo y con la perspectiva de incorporar un recurso alternativo para el control de los mosquitos $A$. aegypti, dentro de un manejo racional de nuestro medio ambiente, la presente investigación tuvo como objetivo evaluar la toxicidad de suspensiones acuosas del extracto etanólico de las semillas, flores, hojas, corteza de ramas y corteza de raíces de $A$. muricata L. «guanábana» sobre larvas del IV estadio de $A$. aegypti L. (Díptera: Culicidae) y sus niveles de susceptibilidad.

\section{Material y métodos}

\section{Obtención y crianza de larvas y adultos de A. aegypti.}

Las larvas de $A$. aegypti se colectaron a partir de una población natural obtenida en el distrito de Trujillo ( $8^{\circ} 08^{\prime} \mathrm{S}$; 79 $04^{\circ} \mathrm{W}$ ), provincia de Trujillo, Región La Libertad; para luego ser trasladadas al Laboratorio de Zoología de la Facultad de Ciencias Biológicas de la Universidad Nacional de Trujillo. La crianza de larvas se realizó según el protocolo de Ventosilla et al. (2001), en tanto que, la crianza de adultos se realizó hasta la quinta generación, según Consoli y Lourenço de Oliveira (1994).

\section{Obtención del material vegetal}

Todo el material vegetal de $A$. muricata se obtuvo del Jardín Botánico de la Facultad de Ciencias Biológicas de la Universidad Na- cional de Trujillo. En el criterio de la toma de las muestras, se consideró la metodología propuesta por Hoss (1992). Luego de lavar y cortar finamente cada parte vegetal, se procedió a secar bajo sombra durante 10 días para, finalmente, realizar su extracción.

\section{Extracción y preparación de las sus- pensiones}

La Figura 1 resume el protocolo de obtención de las suspensiones acuosas del material vegetal, según Lock (1994) con algunas variaciones.

\section{Bioensayos}

Las concentraciones y el desarrollo de los bioensayos, se llevaron a cabo según la metodología propuesta por McLaughlin et al. (1998)

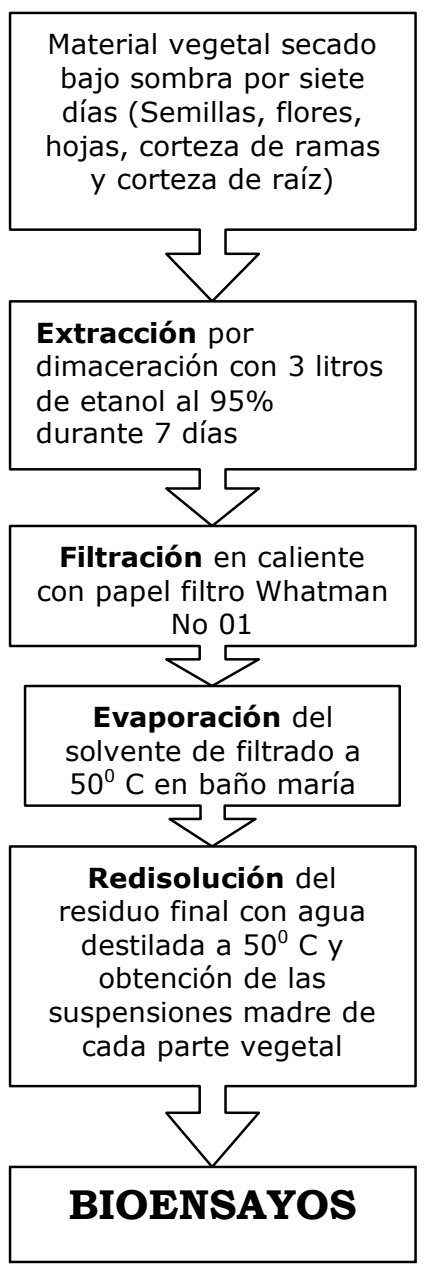

Figura 1. Marcha fitoquímica preparativa de las suspensiones del material vegetal de $A$. muricata L. "guanábana» 
y la WHO (1981), respectivamente. No obstante, previamente se realizaron bioensayos rápidos (Ventosilla et al., 2001), para probar la efectividad de las concentraciones, y luego, en los bioensayos estándar, se tomaron en cuenta las consideradas en la Tabla 1. Se utilizaron 3000 larvas del IV estadio de $A$. aegypti cuya distribución se realizó considerando un sistema por cada parte vegetal. Los sistemas, con sus correspondientes grupos testigo a base de agua destilada, se subdividieron en 6 grupos experimentales, agregándoles las respectivas concentraciones de cada suspensión hasta los $250 \mathrm{~mL}$ de capacidad, con 25 larvas por cuatro repeticiones. Todos los gru- pos se mantuvieron con un rango de temperatura de 23 a $26^{\circ} \mathrm{C}$ y humedad relativa de 75 a $80 \%$. Las lecturas de mortalidad se realizaron a la 12, 24, 36 y 48 horas. Las larvas se consideraban muertas cuando no reaccionaban al momento de ser tocadas con un puntero punta roma en la región cervical (Consoli y Lourenço de Oliveira, 1994).

\section{Análisis estadístico}

Las concentraciones letales al 50\% $\left(\mathrm{CL}_{50}\right)$ y $90 \%\left(\mathrm{CL}_{90}\right)$ y sus limites de confianza, se determinaron utilizando el Epa Probit Analysis Program Ver. 1.5 (Ventosilla et al., 2001). El análisis de varianza para un diseño de medi-

Tabla 1. Porcentajes de mortalidad de cada suspensión sobre larvas del IV estadio de $A$. aegypti a $12,24,36$ y 48 horas.

\begin{tabular}{|c|c|c|c|c|c|}
\hline \multirow[t]{2}{*}{ Suspensiones } & \multirow{2}{*}{$\begin{array}{l}\text { Concentraciones } \\
(\mathrm{mg} / \mathrm{mL})\end{array}$} & \multicolumn{4}{|c|}{ Tiempo (h) } \\
\hline & & 12 & 24 & 36 & 48 \\
\hline \multirow{8}{*}{ Semillas } & 0,005 & 0 & 2 & 5 & 10 \\
\hline & 0,01 & 2 & 7 & 16 & 31 \\
\hline & 0,05 & 25 & 38 & 53 & 73 \\
\hline & 0,1 & 35 & 58 & 75 & 87 \\
\hline & 0,5 & 64 & 100 & 100 & 100 \\
\hline & 1 & 94 & 100 & 100 & 100 \\
\hline & 0,1 & 0 & 0 & 0 & 0 \\
\hline & 0,5 & 0 & 3 & 3 & 3 \\
\hline \multirow{4}{*}{ Flores } & 1 & 3 & 4 & 12 & 18 \\
\hline & 5 & 3 & 15 & 29 & 42 \\
\hline & 10 & 25 & 90 & 94 & 100 \\
\hline & 50 & 78 & 96 & 100 & 100 \\
\hline \multirow{7}{*}{ Hojas } & 0,5 & 0 & 0 & 0 & 0 \\
\hline & 1 & 0 & 1 & 2 & 4 \\
\hline & 5 & 2 & 3 & 6 & 8 \\
\hline & 10 & 31 & 52 & 71 & 79 \\
\hline & 50 & 67 & 82 & 93 & 95 \\
\hline & 100 & 86 & 94 & 100 & 100 \\
\hline & 0,5 & 0 & 0 & 0 & 0 \\
\hline \multirow{5}{*}{$\begin{array}{l}\text { Corteza } \\
\text { ramas }\end{array}$} & 1 & 0 & 0 & 0 & 0 \\
\hline & 5 & 0 & 3 & 10 & 16 \\
\hline & 10 & 6 & 11 & 21 & 29 \\
\hline & 50 & 22 & 52 & 65 & 74 \\
\hline & 100 & 34 & 78 & 87 & 93 \\
\hline \multirow{6}{*}{$\begin{array}{l}\text { Corteza } \\
\text { raíz }\end{array}$} & 0,1 & 0 & 0 & 0 & 0 \\
\hline & 0,5 & 0 & 0 & 0 & 0 \\
\hline & 1 & 0 & 0 & 0 & 0 \\
\hline & 5 & 0 & 0 & 0 & 0 \\
\hline & 10 & 0 & 0 & 0 & 0 \\
\hline & 50 & 2 & 10 & 19 & 34 \\
\hline testigo & absoluto & 0 & 0 & 0 & 0 \\
\hline
\end{tabular}


das de observación repetidas (Darrell, 1975), las rectas probit-logarítmicas y pendientes, para hallar los niveles de susceptibilidad, se realizaron mediante el programa Excel for Windows 2003.

\section{Resultados}

Las diez concentraciones empleadas en la evaluación larvaria de cada parte vegetal abarcaron una mínima de $0,005 \mathrm{mg} / \mathrm{mL}$ a una máxima de $100 \mathrm{mg} / \mathrm{mL}$. En la suspensión de semillas se registraron valores de mortalidad relativamente altos en relación con los demás sistemas, alcanzando un $100 \%$ de mortalidad a las 24 horas a $0,5 \mathrm{mg} / \mathrm{mL}$., mientras que en flores y hojas se observan ambos a las 36 horas a $50 \mathrm{mg} / \mathrm{mL}$ y $100 \mathrm{mg} / \mathrm{mL}$. En la corteza de ramas y raíces, cuyas concentraciones comparativamente altas con las semillas, no registraron $100 \%$ de mortalidad. De oto lado, los valores de mortalidad inicial evidenciaron un $2 \%$ a las 24 horas a $0,005 \mathrm{mg} / \mathrm{mL}$ en semillas, en tanto que en flores, hojas y corteza de ramas muestran un $3 \%$ a $0,5 \mathrm{mg} / \mathrm{mL}, 1 \%$ a $1 \mathrm{mg} / \mathrm{mL}$ y $3 \%$ a $5 \mathrm{mg} / \mathrm{mL}$, respectivamente; $y$ en corteza de raíces se observa una menor mortalidad con un $2 \%$ a las 12 horas a $50 \mathrm{mg} / \mathrm{mL}$. En todos los casos la mortalidad en el grupo testigo fue $0 \%$.

Los rangos de valores de concentraciones letales al $50 \%$ y $90 \%$ con sus límites de confianza y confiabilidad al $95 \%$, se muestran en la Tabla 2. Tal como se observa, las suspensiones de las semillas indican un patrón de efectividad muy superior comparado a las demás partes vegetales. Por ejemplo, a las 12 y 48 horas, el control del $50 \%$ de las larvas se logró con $0,18 \mathrm{mg} / \mathrm{mL}$ (LC $0,10-0,32 \mathrm{mg} / \mathrm{mL}$ ) y $0,02 \mathrm{mg} / \mathrm{mL}$ (LC $0,01-0,026 \mathrm{mg} / \mathrm{mL}$ ), en tanto que el control del $90 \%$ se consiguió con 1,21 $\mathrm{mg} / \mathrm{mL}$ (LC $0,60-4,52 \mathrm{mg} / \mathrm{mL}$ ) y $0,11 \mathrm{mg} / \mathrm{mL}$ (LC 0,08-0,15 mg/mL), respectivamente. En oposición, la suspensión de la corteza de ramas alcanzó valores de $\mathrm{CL}_{50}$ muy superiores, del orden de 232,38 mg/mL (LC 136,77-728,91 $\mathrm{mg} / \mathrm{mL}$ ) y $19,21 \mathrm{mg} / \mathrm{mL}$ (LC $15,95-23,10 \mathrm{mg} /$ $\mathrm{mL}$ ), mientras que la $\mathrm{CL}_{90}$ se obtuvo con $307,65 \mathrm{mg} / \mathrm{mL}$ (LC $202,93-460,15 \mathrm{mg} / \mathrm{mL}$ ) y
Tabla 2. Concentraciones letales al $50 \%\left(\mathrm{CL}_{50}\right)$ y $90 \%\left(\mathrm{CL}_{90}\right)$, limites de confianza $(\mathrm{LC})$ y pendientes (b) de cada suspensión sobre larvas del IV estadio de $A$. aegypti a las $12,24,36$ y 48 horas

\begin{tabular}{|c|c|c|c|c|}
\hline \multicolumn{3}{|c|}{$\begin{array}{l}\text { Suspensión } \\
\qquad(\mathrm{mg} / \mathrm{mL} .) \text { Valor }\end{array}$} & \multirow{2}{*}{$\frac{\text { LC }}{12 \text { horas }}$} & \multirow[t]{2}{*}{ b } \\
\hline & & & & \\
\hline \multirow[t]{2}{*}{ Semillas } & CL50 & 0,18 & $0,10-0,32$ & 2,1 \\
\hline & CL90 & 1,21 & $0,60-4,52$ & \\
\hline \multirow[t]{2}{*}{ Flores } & CL50 & 22,58 & $11,60-77,43$ & 1,3 \\
\hline & CL90 & 101,9 & $40,67-293,33$ & \\
\hline \multirow[t]{2}{*}{ Hojas } & CL50 & 26,86 & $17,08-43,76$ & 2,1 \\
\hline & CL90 & 123 & $68,80-363,00$ & \\
\hline \multirow{5}{*}{$\begin{array}{l}\text { Corteza } \\
\text { ramas } \\
\text { Corteza } \\
\text { raíces } \\
\end{array}$} & CL50 & 232,38 & $136,77-728,91$ & 1,3 \\
\hline & CL90 & 307,65 & $202,93-460,15$ & \\
\hline & CL50 & - & - & - \\
\hline & CL90 & - & - & \\
\hline & & & 24 horas & \\
\hline \multirow[t]{2}{*}{ Semillas } & CL50 & 0,06 & $0,05-0,07$ & 2,5 \\
\hline & CL90 & 0,27 & $0,21-0,36$ & \\
\hline \multirow[t]{2}{*}{ Flores } & CL50 & 6,38 & $1,29-42,96$ & 2,1 \\
\hline & CL90 & 23,89 & $9,17-161,65$ & \\
\hline \multirow[t]{2}{*}{ Hojas } & CL50 & 15,79 & $7,88-32,26$ & 2,3 \\
\hline & CL90 & 65,98 & $32,29-362,24$ & \\
\hline \multirow{5}{*}{$\begin{array}{l}\text { Corteza } \\
\text { ramas } \\
\text { Corteza } \\
\text { raíces }\end{array}$} & CL50 & 43,62 & $36,60-52,63$ & 1,8 \\
\hline & CL90 & 192,82 & $143,76-285,4$ & \\
\hline & CL50 & - & - & - \\
\hline & CL90 & - & - & \\
\hline & & & 36 horas & \\
\hline \multirow[t]{2}{*}{ Semillas } & CL50 & 0,04 & $0,03-0,047$ & 2,2 \\
\hline & CL90 & 0,19 & $0,15-0,25$ & \\
\hline \multirow[t]{2}{*}{ Flores } & CL50 & 4,41 & $1,52-12,90$ & 2,4 \\
\hline & CL90 & 16,35 & $7,10-479,52$ & \\
\hline \multirow[t]{2}{*}{ Hojas } & CL50 & 9,76 & $3,98-26,75$ & 2,7 \\
\hline & CL90 & 30,8 & $14,71-83,51$ & \\
\hline \multirow{4}{*}{$\begin{array}{l}\text { Corteza } \\
\text { ramas } \\
\text { Corteza } \\
\text { raíces }\end{array}$} & CL50 & 27,32 & $22,73-32,99$ & 2 \\
\hline & CL90 & 139,31 & $103,9-204,6$ & \\
\hline & CL50 & - & - & - \\
\hline & CL90 & - & - & \\
\hline & & & 48 horas & \\
\hline \multirow[t]{2}{*}{ Semillas } & CL50 & 0,02 & $0,01-0,026$ & 2 \\
\hline & CL90 & 0,11 & $0,08-0,15$ & \\
\hline \multirow[t]{2}{*}{ Flores } & CL50 & 3,33 & $1,22-8,95$ & 2,6 \\
\hline & CL90 & 12,16 & $5,42-204,74$ & \\
\hline \multirow[t]{2}{*}{ Hojas } & CL50 & 8,25 & $2,42-30,60$ & 2,6 \\
\hline & CL90 & 26,87 & $11,92-52,67$ & \\
\hline \multirow{4}{*}{$\begin{array}{l}\text { Corteza } \\
\text { ramas } \\
\text { Corteza } \\
\text { raíces }\end{array}$} & CL50 & 19,21 & $15,95-23,10$ & 2,1 \\
\hline & CL90 & 97,23 & $73,78-139,23$ & \\
\hline & CL50 & - & - & - \\
\hline & CL90 & - & - & \\
\hline
\end{tabular}

$97,23 \mathrm{mg} / \mathrm{mL}$ ( $\mathrm{LC} 73,78-139,23 \mathrm{mg} / \mathrm{mL}$ ). La ausencia de concentraciones en el caso de la suspensión de la corteza de raíces, no pudieron procesarse adecuadamente por el programa probit, por no registrar por lo menos tres va- 
lores de mortalidad para los cuatro tiempos de evaluación, salvo únicamente a las 48 horas.

Las Figuras 2 a-e denotan la proporción de mortalidad larvaria en unidades probit por el logaritmo de sus respectivas concentraciones, desarrollados hasta las 48 horas con intervalos de 12 horas de evaluación. Todos los gráficos a excepción del último, muestran un patrón de respuesta homogéneo sobre las concentraciones de las suspensiones, según los valores de las pendientes (Montada et al. 1989; Lagunes y Vásquez, 1994) de la Tabla 2. Asimismo, los análisis de varianza en el que se determinaron el efecto de cada variable y su interacción, denotaron diferencias significati-
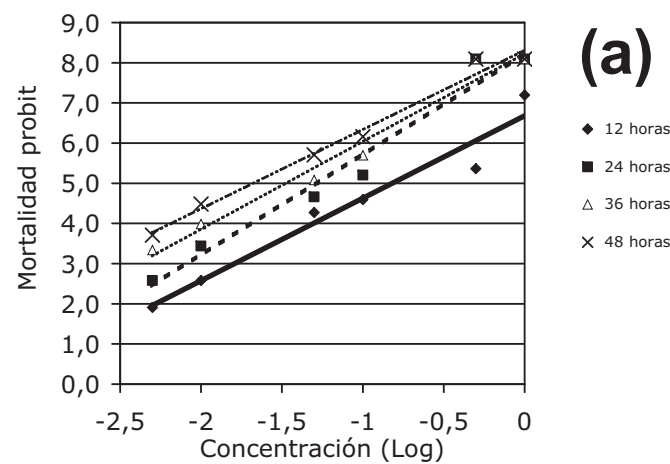

- 24 horas

$\triangle 36$ horas

$\times 48$ horas
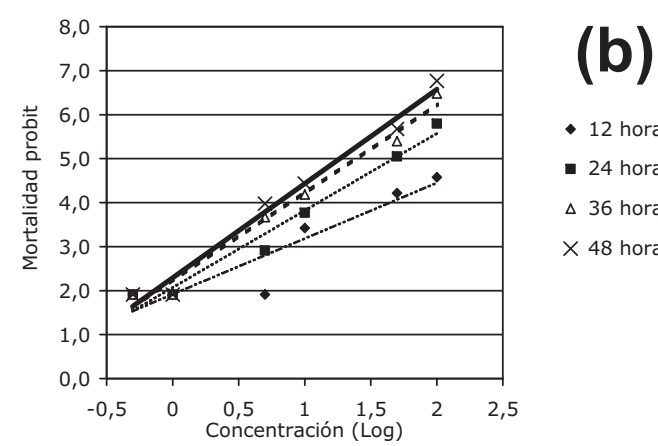

La ventaja en el uso de extractos vegetales como fuentes de una complejidad de moléculas que muestran en conjunto diversas bioactividades, es que los niveles de toxicidad son muy elevados en relación a la acción de compuestos individuales químicamente puros y con el riesgo de generar resistencia. Últimamente se están sumando esfuerzos en la evaluación de insecticidas naturales a partir de extractos heterogéneos de toda la biomasa

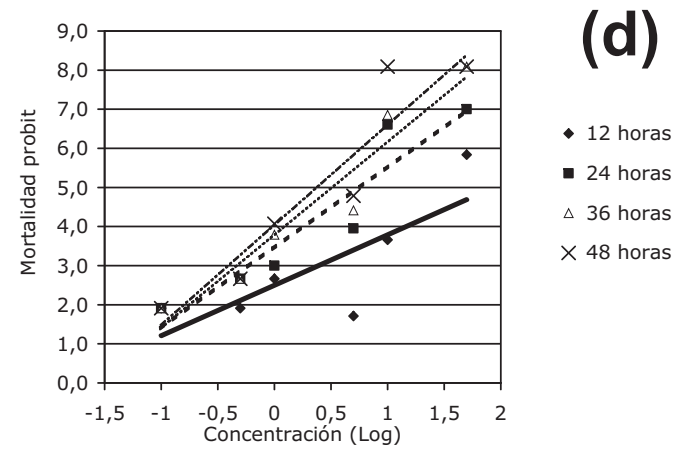

(e)

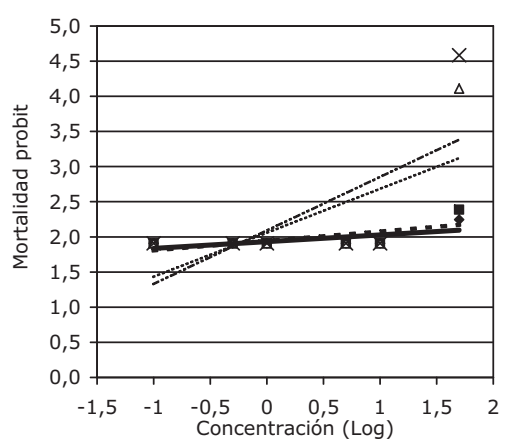

- 12 horas

- 24 horas

$\triangle 36$ horas

$\times 48$ horas
Figura 2. Mortalidad probit por el logaritmo de las concentraciones a las 12, 24, 36 y 48 horas empleando la suspensión acuosa de A. muricata "guanábana»: (a) Semillas, (b) Flores, (c) Hojas, (d) Corteza de ramas y (e) Corteza de raíces 
Tabla 3. Análisis de varianza de un diseño de medidas de observación repetidas y valores de probabilidad estadística para la evaluación de la mortalidad de larvas del IV estadio de A. aegypti.

$$
\begin{aligned}
& \text { Valor de probabilidad } \\
& p<0.05 \text { diferencias significativas } \\
& p>0.05 \text { diferencias no significativas }
\end{aligned}
$$

Suspensiones de:

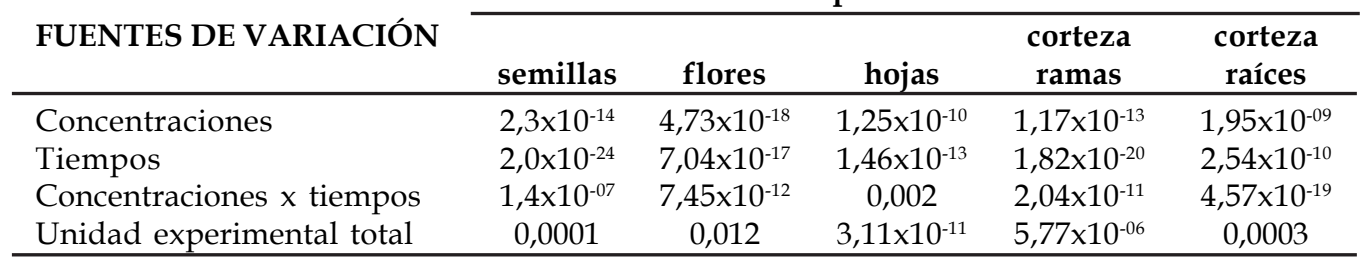

de una planta, con el propósito de producir efecto sinérgico sobre algún organismo blanco (McLaughlin et al., 1998; Leatemia e Isman, 2004) y sumar mayor toxicidad.

Entre las plantas que han destacado por su efecto nocivo sobre larvas de mosquitos mediante extractos orgánicos y no orgánicos, se encuentra las del género Annona, tales como A. bullata, A. glabra, A. muricata y $A$. squamosa, tóxicas a larvas de $A$. aegypti y $A$. cherimola activa contra $A$. aegypti y Anopheles sp. (Bobadilla et al., 2002; Pérez et al., 2004). Dicha actividad larvicida, se produce gracias a un grupo de fitoconstituyentes distribuidos amplia o restringidamente en todas las partes vegetales de las annonaceas (Ratnayaque et al., 1993), causantes de la toxicidad de larvas de $A$. aegypti, aunque Jacobson (1958) y Rodríguez (2000) reportan el control en otros insectos de importancia en salud pública. Padmaja et al. (1995) demostraron con el ácido kaur-16-en-19-oico de la corteza del tallo de $A$. glabra perteneciente a diterpenos kauranos (Valencia, 1995). De igual manera Bruneton (1991) con un complejo de alcaloides benciltetrahidroisoquinoleínico (BTHIQ) de efectos neurotóxicos, y Alali et al. (1998) con un amplio grupo de acetogeninas a través de un mecanismo de inhibición de la NADH ubiquinona oxidoreductasa del complejo I mitocondrial de la cadena respiratoria, presentes en mamíferos e insectos, que reducirían los niveles de ATP desacoplando ciertos mecanismos de resistencia (Alali et al., 1999).
Una característica importante demostrada en grupos representativos de esta familia es la presencia de flavonoides en flores (Valencia, 1995) y hojas (Santos y Salatino, 2000), cuya composición fenólica e hidroxilada, producirían actividad insecticida (Kotkar, 2002) mediante su unión a sistemas enzimáticos (Valencia, 1995), muy similares a los involucrados en la cadena respiratoria, causando potente inhibición. Tales fitoquímicos, sin embargo, suelen distribuirse en tejidos muertos como las cortezas de ramas y raíces y producir efectos similares. De allí la explicación de la baja mortalidad en tales partes, evidenciada probablemente por una distribución restringida de alcaloides, ácidos kaurenóicos y acetogeninas.

Las líneas de regresión dosis-respuesta, en nuestro caso concentración respuesta, muestran la relación existente entre la concentración (en logaritmo) y la proporción de individuos que responden (en unidades probit) con un efecto cuantificable (WHO, 1978). En ellas, cada individuo de la población tiene una determinada tolerancia, que requiere una cierta concentración antes de responder con un efecto. En principio, existe una concentración baja frente a la cual nadie responde, y una concentración alta frente a la cual todos lo hacen (Hickman et al., 2001). La tendencia de las rectas probit-logarítmicas en los cuatro tiempos de evaluación, evidencian dicho patrón, lo que confirma una buena susceptibilidad de las suspensiones sobre las larvas aun cuando las concentraciones hayan variado según la parte 
vegetal evaluada.

La susceptibilidad se producirá cuando la toxicidad, concentración, severidad e incidencia de las suspensiones se incrementen en relación directa al grado o tiempo de exposición (Hickman et al., 2001). Es decir, las sensibilidades de los individuos, que están relacionadas sus variaciones genético-fisiológicas, dependerán de las diferencias en la absorción, metabolismo y excreción de la sustancia o sus metabolitos, así como con la especie o partes del vegetal, preparación, extracción y aplicación de la sustancia insecticida (Lagunas y Vásquez, 1994) que anticiparían la variación de la mortalidad, según los resultados de la Tabla 1 y de del análisis de varianza (Tabla 3 ). De la misma manera los valores de las $\mathrm{CL}_{50}$, $\mathrm{CL}_{90}$ (Tabla 2) mostrarían dicha tendencia, requiriéndose de mayor suspensión para producir mayor efecto tóxico en menor tiempo de exposición y viceversa.

Todas las suspensiones mostraron toxicidad larvicida. Los resultados favorecen a la suspensión acuosa de las semillas, por ser un órgano de reserva con mayor probabilidad de contener los principios activos de $A$. muricata, en comparación a la corteza de raíces cuya toxicidad fue muy baja. La mortalidad varió en función a la parte vegetal, proporción de principios activos y variación de concentraciones.

\section{Literatura citada}

Alali F., W. Kaakeh, G. Bennett \& McLaughlin, J. 1998. Annonaceous acetogenins as natural pesticides: Potent toxicity against insecticide-suceptible and-resistant German cockroaches (Dictyoptera: Blattellidae) J. Econ. Entomol. 91(3): 641-649

Alali F., X. Liu \& J. McLaughlin. 1999. Annonaceous acetogenins: Recent progress. J Nat Prod. 62(3):504-540

Bobadilla M., G. Zavaleta, F. Gil, L. Pollack y M. Sisniegas. 2002. Efecto bioinsecticida del extracto etanólico de las semillas de Annona muricata Miller «chirimoya» y A. muricata Linneaus «guanábana» sobre larvas del IV estadio de Anopheles sp. Revista Peruana de Biología 9(2): 64-73.
Bruneton J. 1991. Elementos de fitoquímica y farmacognosia. Edit. Acribia. Zaragoza. España.

Consoli, R. \& R. Lourenço de Oliveira. 1994. Principais mosquitos de importancia sanitária no Brasil. Edit. Fiocruz. Rio de Janeiro. Brasil.

Darrell, R. 1975. Multivariate Statical Methods in Behavioral Research. Edit. Mc Graw-Hill Book Company.

Hickman R, V. Robert y D. Murdoch. 2001. Manual de Evaluación y Manejo de Sustancias Tóxicas en Aguas Superficiales. Sección 2. Evaluación y Manejo del Riesgo. OPS/CEPIS/PUB/01.66

Hoss R. 1992. Cuaderno de Trabajo 1. Guía Metodológica: Uso de Extractos Vegetales en la Regulación de Plagas. Red de Acción de Alternativas al Uso de Agroquímicos. Lima. Perú.

Jacobson M. 1958. Insecticides from plants. A review of the literature (1941-1953). Agriculture Mined Book N ${ }^{\circ}$ 154. U.S. Government Printing Office. Washington. USA.

Kotkar H., P. Mendki, S. Sadan, S. Jha, S. Upasani \& V. Maheshwari. 2002. Antimicrobial and pesticidal activity of partially purified flavonoids of Annona squamosa. Pest Manag Sci. 58(1): 33-37.

Lagunes A. y M. Vásquez. 1994. El bioensayo en el manejo de insecticidas y acaricidas. Colegio de Post graduados en Ciencias Agrícolas. Montecillo. México.

Leatemia J. \& B. Isman. 2004. Toxicity and antifeedant activity of crude seed extracts of Annona squamosa (Annonaceae) against lepidopteran pests and natural enemies. International J Trop Insect Science. 24(1):150-158

Lock O. 1994. Investigación fitoquímica. Métodos en el estudio de productos naturales. 2. ed. Fondo Editorial de la Pontificia Universidad Católica del Perú. Lima. Perú.

Mathur S. 2003. Prospects of using herbal products in the control of mosquito vectors. Bull Indian Council Med Res. 33(1)1-10.

McLaughlin J., R. Lingling \& A. Jon. 1998. The use of biological assays to evaluate botanicals. Drug Information Journal. 32 (513-524).

Montada D., R. Tang, A. Navarro y F. García. 1989. Estudio de la Sensibilidad al Dimilín (Diflubenzurón) en una cepa de Aedes aegypti Linneaus, 1762 y de Culex quinquefasciatus Say, 1823 criadas en Laboratorio. Rev Cub. Med. Trop. 41(1): 56-63.

OMS. 1992. Resistencia de los Vectores de Enfermedades a los Plaguicidas. $15^{\circ}$ Informe del Comité de Expertos de la OMS en Biología de Vectores y Lucha Antivectorial (Serie de Informes Técnicos $\left.N^{\circ} 818\right)$. Ginebra. Suiza.

OPS. 2001. Situación de los programas de malaria en las Américas. Bol Epid Org Pan Salud. 22(1):10-14 
Padmaja V., V. Thankamany, N. Hara, Y. Fujimoto, A. Hisham. 1995. Biological activities of Annona glabra. J Ethnopharmacol. 48(1):21-24

Pérez R., C. Rodríguez, J. Lara, R. Montes y G. Ramírez. 2004. Toxicidad de aceites, esencias y extractos vegetales en larvas de mosquito Culex quinquefasciatus say (Diptera: Culicidae). Acta Zoológica Mexicana 20(1): 141-152.

Ratnayake, S., J.K. Rupprecht, W.M. Potter, \& J.L. McLaughlin. 1993. Evaluation of the pawpaw tree, Asimina triloba (Annonaceae), as a commercial source of the pesticidal annonaceous acetogenins. p. 644-648. In: J. Janick and J.E. Simon (eds.), New crops. Wiley, New York.

Rodríguez C. 2000. Plantas Contra Plagas: Potencial práctico de ajo, anona, nim, chile y tabaco. Edit. de la RAPAM. México. D.F. México.

Rose R. 2001. Pesticide and Public Health: Integrated Methods of Mosquito Management. Emerg Infect Dis 7(1):17-23.

Santos D. \& M. Salatino. 2000. Foliar flavonoids of Annonaceae from Brazil: taxonomic significance. Phytochemistry. 55:567-573.
Valencia C. 1995. Fundamentos de fitoquímica. Edit. Trillas. México DF. México.

Ventosilla P., B. Infante, J. Merello y J. Chauca. 2001. Guía de Prácticas para la Producción de Bacillus thuringiensis subsp. israelensis usando alternativas locales para el control de vectores de enfermedades. OPS/OMS/ROW/IMTAvH. Universidad Peruana Cayetano Heredia.

Weinzierl R. \& T. Henn. 1992. Alternatives in insect management: Biological and biorational approaches. North Central Regional Extension Publication 401. University of Iliinois at Urbana-Champaign

WHO. 1978. Principles and methods for evaluating the toxicity of chemicals. Part I. Environmental health Criteria No. 6. World Health Organization, Geneva

WHO. 1981. Instructions for determining the susceptibility or resistance of mosquito larvae to insecticides. WHO/VBC/81.807.

Zimmerman R. 2001. Control selectivo de vectores y el rol de Bti en el control de vectores. En Guía de Prácticas para la Producción de Bacillus thuringiensis subsp. israelensis usando alternativas locales para el control de vectores de enfermedades. OPS/OMS/ROW/IMTAvH. 